\title{
Trophoblast Differentiation Modulates the Activity of Caspases in Primary Cultures of Term Human Trophoblasts
}

\author{
KAMRAN YUSUF, STEVEN D. SMITH, YOEL SADOVSKY, AND D. MICHAEL NELSON \\ Departments of Pediatrics, Division of Newborn Medicine (K.Y.) and Obstetrics and Gynecology (S.D.S., \\ Y.S., D.M.N.), and Cell Biology and Physiology (Y.S.), Washington University School of Medicine, \\ St. Louis, Missouri 63110-1094
}

\begin{abstract}
ABSTRA
Cultured human cytotrophoblasts are more susceptible than
syncytiotrophoblasts to hypoxia-induced apoptosis. Caspases are
cysteine proteases that cleave cellular components to effect the
apoptotic cascade. We hypothesized that cultured cytotropho-
blasts exhibit a higher activity of caspases when compared with
syncytiotrophoblasts. Using western analysis, we demonstrated
that the pro-caspases $3,6,8$, and 9 are expressed in cytotropho-
blasts cultured for 24 h, and also, in trophoblasts cultured $72 \mathrm{~h}$
when syncytiotrophoblasts have formed. Importantly, we found
significantly higher activity of all four caspases in trophoblasts
cultured 24 h compared with cells cultured $72 \mathrm{~h}$. Colchicine and
DMSO, which hinder trophoblast differentiation, enhanced the
activity of all four caspases in cells cultured $72 \mathrm{~h}$. Conversely,
caspase activity was reduced in trophoblasts cultured for $24 \mathrm{~h}$ in
the presence of epidermal growth factor, which enhances differ-
\end{abstract}
Human placental villi are covered by terminally differentiated, multinucleated syncytiotrophoblasts that are in direct contact with maternal blood. The subjacent mononucleated cytotrophoblasts provide a proliferative, stem cell population that differentiates and fuses to replenish the syncytium. Turnover of the trophoblast bi-layer occurs, in part, through apoptosis $(1,2)$. The balance of proliferation, differentiation, and apoptosis in the trophoblast layer of villi ultimately determines the mass of functional trophoblast available to regulate nutrient and waste exchange between the maternal and fetal circulations. Preeclampsia and fetal growth restriction (FGR) are associated with placental dysfunction, including villous hypoxia, altered differentiation and enhanced apoptosis in trophoblast (3-6). We previously showed that hypoxia hinders differentiation (7) and enhances apoptosis (8) in cultured

October 3, 2001; January 29, 2002.

Correspondence and reprint requests: D. Michael Nelson, M.D., Ph.D., Department of Obstetrics/Gynecology, Washington University School of Medicine, 4566 Scott Ave, St. Louis, MO, 63110-1094; E-mail: nelsondm@msnotes.wustl.edu

Supported by NIH Grant HD 29190.

DOI: 10.1203/01.PDR.0000022346.66626.02 entiation. This effect was most pronounced on caspase 3 and was attenuated by addition of the tyrosine kinase inhibitor AG1478. We conclude that cytotrophoblasts exhibit a higher activity of caspases $3,6,8$, and 9 when compared with the more differentiated syncytium. This may account for the higher susceptibility of cytotrophoblasts to hypoxia-induced apoptosis. (Pediatr Res 52: 411-415, 2002)
EGF, epidermal growth factor
EGFR, epidermal growth factor receptor
FGR, fetal growth restriction
M 199, medium 199
cRFU, corrected relative fluorescent units

trophoblasts from term placentas. Importantly, we found that the cytotrophoblasts were more susceptible than syncytiotrophoblasts to hypoxia-induced apoptosis.

The regulation of apoptotic cell death is complex (9). Multiple ligand-receptor interactions and diverse stimuli that modulate mitochondrial function converge to enhance the activity of initiator caspases, cysteine proteases that self-amplify their enzymatic activity when stimulated. These enzymes activate downstream effector caspases that cleave membrane, cytoplasmic, and nuclear components, effectively dismantling the cell. Caspases are well known for their role in cell suicide by apoptosis, but recent studies indicate they also play a role in cell proliferation and differentiation (10-12). Knowledge of the expression and activity of caspases in placental villi and cultured trophoblasts is in its infancy (13-15). The proforms of the initiator caspase 8 and the effector caspases 3, 6, and 7 are expressed in villous cytotrophoblast and syncytiotrophoblasts. Indirect evidence from immunohistochemical studies suggests that the fully active forms of these enzymes may be differentially expressed in both cytotrophoblast and syncytiotrophoblast on placental villi. We chose to examine two initiator 
caspases, 8 and 9, and two effector caspases, 3 and 6, because they are pivotal in both the ligand-receptor and the mitochondrial pathways to apoptotic cell death $(16,17)$. We tested the hypothesis that trophoblast differentiation alters the basal activities of caspases in primary cultures of human trophoblasts from term placental villi.

\section{MATERIALS AND METHODS}

Cell isolation and culture. Informed consent for the use of human placentas was obtained by a protocol approved by the Institutional Review Board of Washington University, School of Medicine. Placentas were obtained immediately after term singleton deliveries following uncomplicated pregnancies. Cytotrophoblasts were isolated by the trypsin-DNase, Percoll (Pharmacia, Piscataway, NJ, U.S.A.) gradient centrifugation method described by Kliman et al. (18). with modifications (8). The cell isolate and culture characteristics in vitro have been previously described $(7,8)$. Control cytotrophoblasts were plated on polystyrene plates (Becton Dickinson, Franklin Lakes, NJ, U.S.A.) in duplicates or triplicates at $3 \times 10^{5}$ cells $/ \mathrm{cm}^{2}$ in medium 199 (Tissue Culture Facility, WA University) with $10 \%$ fetal bovine serum (Hyclone, Logan, UT, U.S.A.) $20 \mathrm{mmol} \mathrm{N}$-[2-hydroxyethyl] piperazine-N-[2ethanesulfonic acid] (HEPES; Sigma Chemical Co., St. Louis MO, U.S.A.), and $2 \mathrm{mmol}$ L-glutamine (Sigma Chemical Co., St. Louis, MO, U.S.A.) in a $5 \%$ carbon dioxide-air atmosphere at $37^{\circ} \mathrm{C}$. Where indicated, cultures were supplemented with one or more of the following compounds: the pan-caspase inhibitor ZVAD-FMK (30 $\mu \mathrm{M}$; Enzyme System Products, Livermore, CA, U.S.A.), colchicine $(250 \mu \mathrm{M}$; Sigma Chemical Co., St. Louis, MO, U.S.A.), DMSO (DMSO; 1.5\% vol/vol; Sigma Chemical Co.), epidermal growth factor (EGF; 100ng/ $\mathrm{mL}$; Upstate Biotechnology, Lake Placid, NY, U.S.A.), or the EGF receptor (EGFR) antagonist AG1478 $(1 \mu \mathrm{M}$; Calbiochem, San Diego, CA, U.S.A.). Media were replaced every $24 \mathrm{~h}$, and supernatants were stored at $-20^{\circ} \mathrm{C}$ until assayed.

Hormone assays. Culture supernatants were assayed for hCG in duplicates by ELISA (DRG Diagnostics, Marburg, Germany) according to the manufacturer's instructions. Values represent mean $( \pm \mathrm{SD}) \mathrm{mIU} / \mathrm{mL}$ of hCG secreted in the previous $24 \mathrm{~h}$.

Expression analysis. Cell lysis, sonication, PAGE and transfer were performed as previously described (8), modified to use $30 \mu \mathrm{g}$ protein per lane and $10 \%$ skim milk for blocking nonspecific proteins. Membranes were incubated overnight at $4^{\circ} \mathrm{C}$ with each of the following primary antibodies diluted 1:100 with TBST: goat polyclonal anti-pro-caspase 3 (Santa Cruz, Santa Cruz, CA, U.S.A.), rabbit polyclonal anti-procaspase 6 (Cell Signaling Technology, Beverly, MA, U.S.A.), goat polyclonal anti-pro-caspase 8 (Santa Cruz), and rabbit polyclonal anti-pro-caspase 9 (Cell Signaling Technology). Subsequently, the blot was incubated for $1 \mathrm{~h}$ with HRPconjugated secondary antibody (Santa Cruz), washed, and processed for luminescence using Amersham ECL kit (Amersham Pharmacia Biotech). The densitometric analysis was performed using a Molecular Dynamics densitometer and Im-
ageQuant v. 3.3 software (Molecular Dynamics, Sunnyvale, CA, U.S.A.).

Caspase Activity Assays. Cells were lysed in buffer containing $10 \mathrm{mM}$ HEPES, $\mathrm{pH} 7.4,7.5 \mathrm{mM} \mathrm{MgCl} 2,1 \%$ Triton X-100, $2 \mathrm{mM}$ EGTA, $1 \mathrm{mM}$ DTT, and protease inhibitor cocktail (Sigma Chemical Co.). Samples were sonicated with one 10 second burst using a sonic dismembrator (Fisher, Pittsburgh, PA, U.S.A.) and centrifuged at $10,000 \mathrm{~g}$ for $20 \mathrm{~min}$. Protein concentration of the resulting supernatant was determined with the Bio-Rad DC protein assay kit (BioRad, Hercules, CA, U.S.A.). Caspase assays were performed in triplicate with 30 $\mu \mathrm{g}$ protein per well and activity was measured in a FluoroCount microplate fluorometer (Packard, Meridan, CT, U.S.A.). This assay quantified the fluorescence that resulted from proteolytic cleavage of caspase specific synthetic peptides linked to 7-amino-4-methylcoumarin (AMC). Once cleaved from the substrate, the AMC emitted fluorescence at $430-460 \mathrm{~nm}$ when excited at $365-380 \mathrm{~nm}$. Assay buffer consisted of $10 \mathrm{mM}$ HEPES, pH 7.4, $50 \mathrm{mM} \mathrm{KCl}, 10 \%$ sucrose, and $3 \mathrm{mM}$ DTT. The lysates were incubated in 96 well plates in the dark at room temperature for $3 \mathrm{~h}$ with one of the following caspase specific substrates in assay buffer: Ac-DEVD-AMC (caspase-3; Anaspec, San Jose, CA, U.S.A.), Ac-VEID-AMC (caspase-6; Calbiochem, San Diego, CA, U.S.A.), Ac-IETD-AMC (caspase-8; Anaspec), and Ac-LEHD-AMC (caspase-9; Anaspec). Background fluorescence from wells without protein was subtracted, and data were expressed as corrected relative fluorescence units (cRFU). There was no difference in caspase activity for cells cultured freshly after isolation compared with cells cultured after freezing (data not shown). We thus used previously frozen cells for our experiments.

Statistical analysis. Data are mean \pm SD. Comparisons were made using $t$ test or one-way ANOVA with Bonferroni correction where multiple comparisons were made. A $p<0.05$ was significant.

\section{RESULTS}

Cells cultured $24 \mathrm{~h}$ under standard conditions were predominantly the less differentiated, mononucleated cytotrophoblasts while cultures at $72 \mathrm{~h}$ exhibited abundant multi-nucleated syncytiotrophoblasts, indicative of morphologic differentiation. The differentiation of the trophoblast cultures was confirmed by the marked rise in the media level of hCG during culture (data not shown), as previously described (7).

We used western analysis to determine expression of procaspases in trophoblasts cultured for $24 \mathrm{~h}$ and for $72 \mathrm{~h}$. The pro-caspases examined included two effector enzymes, 3 and 6 , and two initiator caspases, 8 and $9(16,17)$. We found that all four pro-caspases were detectable in trophoblasts at both $24 \mathrm{~h}$ and $72 \mathrm{~h}$ of culture (Fig. 1). Pro-caspase 3 showed a 2-fold higher level of expression in cells cultured $24 \mathrm{~h}$ compared with those cultured $72 \mathrm{~h}$. There was no difference in relative expression for pro-caspases 6,8 , and 9 between the twotrophoblast phenotypes.

We next examined the activity of caspases $3,6,8$, and 9 in trophoblasts cultured under standard conditions for $24 \mathrm{~h}$ and $72 \mathrm{~h}$. The activity of each caspase was significantly higher in 


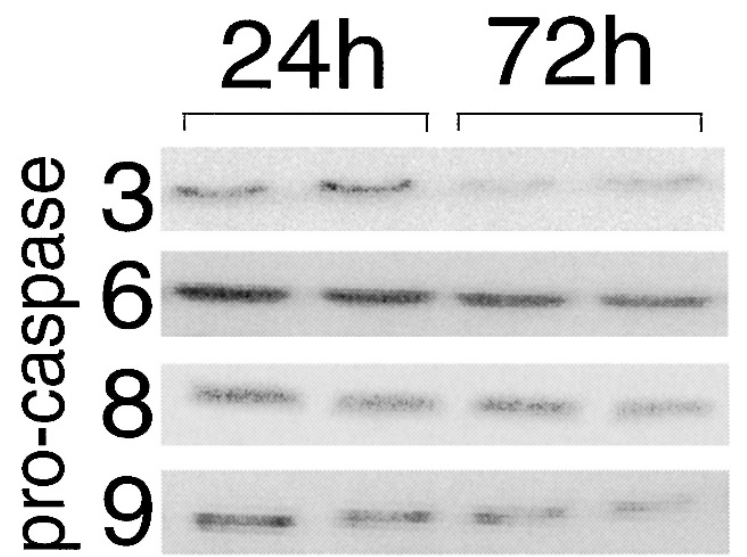

Figure 1. Expression of pro-caspases at $24 \mathrm{~h}$ and $72 \mathrm{~h}$ in primary trophoblasts cultured in medium 199, as shown by western blot. Time points are represented by duplicate wells from one placenta. Densitometric analysis revealed a 2-fold higher pro-caspase 3 expression in cells at $24 \mathrm{~h}$ compared with $72 \mathrm{~h}$. ( $p=$ $0.03)$. There was no significant difference in expression for the other three pro-caspases in cells cultured $24 \mathrm{~h}$ compared with $72 \mathrm{~h}$. The western blot is representative of four independent primary trophoblast cultures.

the less differentiated cells at $24 \mathrm{~h}$ compared with the more differentiated cells at $72 \mathrm{~h}$ (Fig. 2A). To verify that the enzymatic cleavage of substrate was effected by caspases, we cultured cells for $30 \mathrm{~min}$ in the presence or absence of the pan-caspase inhibitor ZVAD-FMK before an additional 24h of culture before assay of caspase activities. The inhibitor significantly reduced the caspase activities to $5 \%$ to $25 \%$ of control levels $(p<0.001$; Fig. 2B). Together, these data indicate that active caspases are expressed in both trophoblast phenotypes, but all four caspases exhibit a lower activity in syncytiotrophoblasts compared with cytotrophoblasts.

To rule out the possibility that time in culture, and not differentiation, diminished caspase activity, we analyzed caspase activity in trophoblast cultures grown $72 \mathrm{~h}$ in standard conditions to trophoblasts grown $72 \mathrm{~h}$ in the presence of either DMSO or colchicine. These chemicals are known to hinder trophoblast differentiation, resulting in cultures at $72 \mathrm{~h}$ with a predominantly cytotrophoblast phenotype $(19,20)$. Using these paradigms, we predicted that cells grown $72 \mathrm{~h}$ in the presence of DMSO or colchicine would have higher caspase activity than the more differentiated trophoblast in native conditions. We verified that a substantially lower media hCG level was present in cells cultured $72 \mathrm{~h}$ in media with DMSO $(1.7 \pm 2.2$ $\mathrm{mIU} \mathrm{hCG/mL})$ or colchicine $(1.5 \pm 2.2 \mathrm{mIU} \mathrm{hCG} / \mathrm{mL})$, compared with control cells in medium 199 alone $(483.9 \pm 12.1$

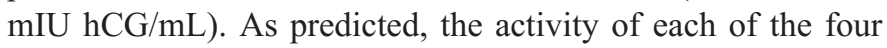
caspases was significantly higher in cytotrophoblasts cultured in DMSO or colchicine for $72 \mathrm{~h}$, compared with untreated cultures of syncytiotrophoblasts at $72 \mathrm{~h}$ (Fig. 3). These results support the previous findings that cytotrophoblasts exhibit a higher caspase activity, compared with the syncytiotrophoblasts.

EGF is known to enhance both morphologic and biochemical differentiation of trophoblasts in culture (21). We cultured cells $24 \mathrm{~h}$ in the presence or absence of EGF and measured hCG levels in medium and caspase activities in cell extracts. As expected $(8,21)$, hCG levels were substantially higher in
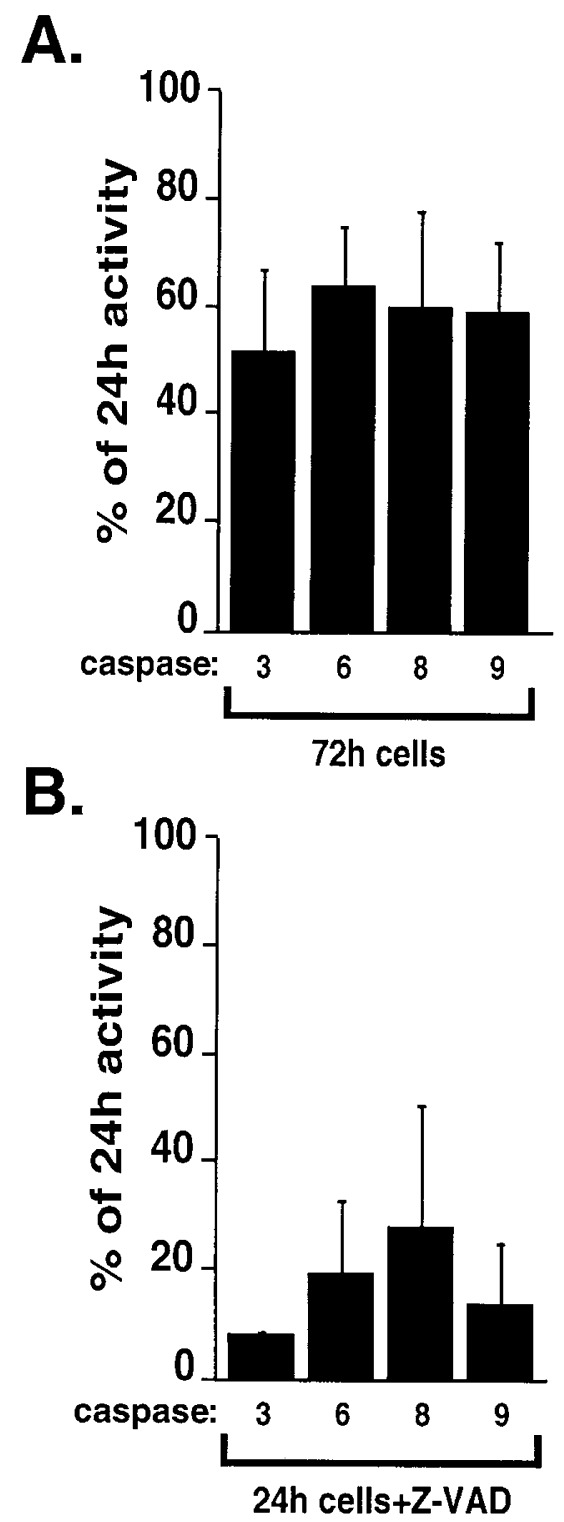

Figure 2. Caspase activity in primary trophoblasts cultured $24 \mathrm{~h}$ or $72 \mathrm{~h} .(A)$ Data (mean $\pm \mathrm{SD}$ ) are activity of each caspase in trophoblasts cultured $72 \mathrm{~h}$, expressed as a percentage of activity in control cells cultured $24 \mathrm{~h}$. The mean caspase activity in cells at 24 hour was $11,688 \mathrm{cRFU}$ for caspase 3, $2525 \mathrm{cRFU}$ for caspase 6, $2848 \mathrm{cRFU}$ for caspase 8, and $1567 \mathrm{cRFU}$ for caspase 9. The activity of each caspase was significantly lower in the $72 \mathrm{~h}$ cells $(p<0.005)$. $(B)$ The pan-caspase inhibitor Z-VAD significantly reduced caspase activity in cells cultured $24 \mathrm{~h}$, compared with control cells without inhibitor $(p<0.001)$. The results are from five independent primary trophoblast cultures.

cultures exposed to EGF $(21.7 \pm 5.6 \mathrm{mIU} \mathrm{hCG} / \mathrm{mL})$, compared with cultures without ligand $(4.8 \pm 5.6 \mathrm{mIU} \mathrm{hCG} / \mathrm{mL})$. Importantly, EGF treated cells showed significantly reduced activity of caspase 3,6 , and 9 at 24 h of culture, compared with control without ligand (Fig. 4A). The EGF effect was most pronounced on the activity of caspase 3 , and this finding was consistent with the above results where caspase 3 showed the most noticeable change in expression (Fig. 1) and activity (Fig. 2). We thus assayed caspase 3 activity in cultures exposed to a range of EGF concentrations in the presence or absence of $A G$ 1478 , a specific inhibitor of EGF receptor tyrosine kinase (22). We found that the highest level of EGF, $(100 \mathrm{ng} / \mathrm{mL})$ maxi- 


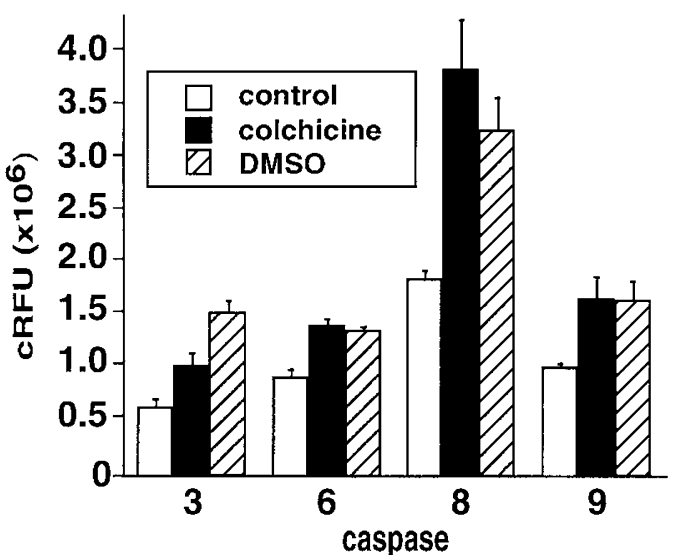

Figure 3. Caspase activity in primary trophoblasts cultured for $72 \mathrm{~h}$ in standard medium (control) or in the presence of colchicine $(250 \mu \mathrm{M})$ or DMSO $(1.5 \%$, vol $/ \mathrm{vol})$. Data (mean $\pm \mathrm{SD})$ are corrected relative fluorescent units, as described in Methods. The activity of each caspase was significantly higher than control in both the colchicine $(p<0.01)$ and the DMSO $(p<$ $0.005)$ paradigms. The results are from five independent primary trophoblast cultures.

mally reduced the caspase activity to $60 \%$ of control values (Fig. 4B). However, caspase 3 activity remained at control levels when AG 1478 was present, with or without EGF additions (Fig. 4B). These results indicate that EGF, a known inducer of trophoblast differentiation, lowers caspase activity in cultured trophoblasts, with the most pronounced effect on caspase 3 . In addition, the effect of EGF on caspase 3 activity in trophoblast is likely mediated through the EGFR.

\section{DISCUSSION}

We studied the role of caspases in trophoblast to gain insight into the contribution of caspases to the differential susceptibility of the two trophoblast phenotypes to apoptotic stimuli. The western analyses demonstrated that both cytotrophoblasts and syncytiotrophoblasts express the four pro-forms of the caspases studied with higher levels of pro-caspase 3 present in cytotrophoblasts. Despite a lack of difference in protein expression of pro-caspases 6,8 , and 9 , the assessment of enzyme activities showed marked differences between the two phenotypes for all caspases evaluated. The highest caspase activity was found in cytotrophoblasts, and caspase activity decreased with trophoblast differentiation. Three lines of evidence support this premise: 1) There was a higher caspase activity in cytotrophoblasts present at $24 \mathrm{~h}$ under standard conditions, compared with cultures at $72 \mathrm{~h}$ that contained differentiated syncytiotrophoblasts. 2) Compared with cultures grown $72 \mathrm{~h}$ in standard conditions, the activity of all four caspases was substantially higher when cells were cultured $72 \mathrm{~h}$ in DMSO (19) or colchicine (20), which are known to hinder differentiation and maintain the cytotrophoblast phenotype. 3) Total caspase activity of cells whose differentiation was enhanced by exposure for $24 \mathrm{~h}$ to EGF was significantly lower than caspase activity in control cells grown $24 \mathrm{~h}$ without ligand. We speculate that modulation of caspase activity in trophoblast may be important in the differentiation process of these cells and may also
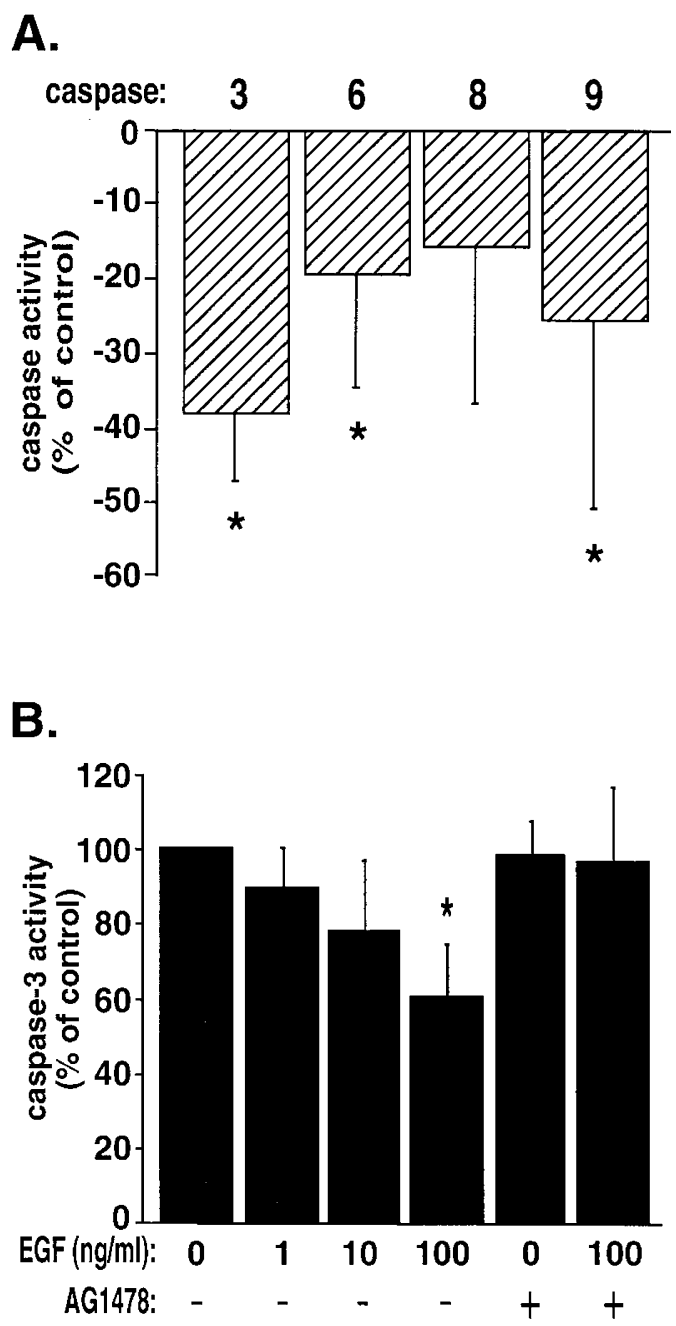

Figure 4. (A) Caspase activity in primary trophoblasts cultured for $24 \mathrm{~h}$ in the absence (control) or presence of EGF $(100 \mathrm{ng} / \mathrm{mL})$. Data are mean $( \pm \mathrm{SD})$ percent of the caspase activity in control cultures from six independent primary trophoblast cultures. Mean caspase activity in control cultures was $6751 \mathrm{cRFU}$ for caspase 3, $3008 \mathrm{cRFU}$ for caspase 6, $3776 \mathrm{cRFU}$ for caspase 8, and 1721 cRFU for caspase 9. The activities of caspases 3, 6, and 9 in cultures with EGF were significantly lower than control $\left({ }^{*} p<0.05\right)$. (B) Caspase 3 activity in trophoblasts cultured for $24 \mathrm{~h}$ in standard medium in the absence (control) or presence of increasing concentrations of EGF, with $1 \mu \mathrm{M}$ AG1478 where indicated. Data are mean $( \pm \mathrm{SD})$ percent of the caspase activity in control cultures. Results are from six independent primary trophoblast cultures. ${ }^{*} p<$ 0.05 compared with control.

contribute to the differential sensitivity to hypoxia (8) exhibited by the two villous trophoblast phenotypes.

Villous trophoblasts undergo morphologic and biochemical differentiation in vivo and in vitro, as mononucleated cytotrophoblasts fuse to form the multinucleated syncytiotrophoblasts, which secrete hCG (23). Apoptosis also occurs in the trophoblast layer of placental villi $(1,2)$, especially at sites of syncytial knots (24). The differentiation and apoptotic pathways in trophoblasts have several features in common, and others have suggested that they are co-regulated as cytotrophoblasts become syncytiotrophoblasts $(13,14)$. For example, phosphatidylserine is exteriorized on the surface membrane of cells during apoptosis (9), but this phospholipid is externalized as part of both the apoptotic and differentiation processes in 
trophoblasts $(13,25)$. Caspases influence differentiation of a variety of cells $(10-12)$. The presence of different enzyme activities in cytotrophoblasts and syncytiotrophoblasts suggests that the regulation of caspase activity may be part of the differentiation program. Alternatively, the higher caspase activities in cytotrophoblasts may be necessary to cleave substrates important to the formation of syncytiotrophoblasts. Further studies are needed to determine what role caspases may play in trophoblast differentiation, independent of their role in apoptosis.

Hypoxia and thromboxane treatment hinder differentiation $(7,26,27)$, and enhance apoptosis in cultured trophoblast $(8$, 27). Importantly, undifferentiated cytotrophoblasts are most susceptible to the apoptosis induced by apoptotic stimuli while the syncytiotrophoblast is relatively resistant $(8,27)$. Our caspase studies point to one mechanism that explains this differential susceptibility. Pro-caspases reside intracellularly in a latent form with enzyme activity of $\leq 2 \%$ of the caspase (16, 17). After cell exposure to an activating stimulus, caspases undergo aggregation and enhanced autocatalytic cleavage that amplifies latent enzyme activity to yield the fully active caspase. Our data suggest that the higher endogenous caspase activity in the cytotrophoblasts sets a lower threshold for these cells to undergo apoptosis when exposed to apoptotic stimuli like hypoxia. Accordingly, the lower endogenous caspase activity in syncytiotrophoblast would make this phenotype less susceptible to apoptosis induced by exogenous stimuli. Preeclampsia and fetal growth restriction are associated with villous hypoxia and enhanced trophoblast apoptosis $(3-6,28)$. If hypoxia induces apoptosis of trophoblast in vivo in a manner similar to cultured trophoblast, drugs that inhibit caspases may provide a therapeutic approach to modulate the deleterious effects of these enzymes. This approach has been recently tested in the brain, where caspase inhibitors reduced the level of apoptosis after exposure to either hypoxic-ischemic or infectious insults $(29,30)$. Further investigation into the role of caspases in trophoblast apoptosis should be revealing.

\section{REFERENCES}

1. Smith SC, Baker PN, Symonds EM 1997 Placental apoptosis in normal human pregnancy. Am J Obstet Gynecol 177:57-65

2. Nelson DM 1996 Apoptotic changes occur in syncytiotrophoblast of human placental villi where fibrin type fibrinoid is deposited at discontinuities in the villous trophoblast. Placenta 17:387-391

3. Kingdom JCP, Kaufmann P 1997 Oxygen and placental villous development: Origins of fetal hypoxia. Placenta 18:613-621

4. Allaire AD, Ballenger KA, Wells SR, McMahon MJ, Lessey BA 2000 Placental apoptosis in preeclampsia. Obstet Gynecol 96:271-276
5. Smith SC, Baker PN, Symonds EM 1997 Increased placenta apoptosis in intrauterine growth restriction. Am J Obstet Gynecol 177:1395-1401

6. Leung DN, Smith SC, To KF, Sahota DS, Baker PN 2001 Increased placental apoptosis in pregnancies complicated by preeclampsia. Am J Obstet Gynecol 184:1249-1250

7. Nelson DM, Johnson RD, Smith SD, Anteby EY, Sadovsky Y 1999 Hypoxia limits differentiation and up-regulates expression and activity of prostaglandin $\mathrm{H}$ synthase 2 in cultured trophoblast from term human placenta. Am J Obstet Gynecol 180:896902

8. Levy R, Smith SD, Chandler K, Sadovsky Y, Nelson DM2000 Apoptosis in human cultured trophoblasts is enhanced by hypoxia and diminished by epidermal growth factor. Am J Physiol Cell Physiol 278:C982-C988

9. Levy R, Nelson DM 2000 To be, or not to be, that is the question. Apoptosis in the human trophoblast. Placenta 21:1-13

10. De Maria R, Zeuner A, Eramo A, Domenichelli C, Bonci D, Grignani F, Srinvasula SM, Alnemri ES, Testa U, Peschle C 1999 Negative regulation of erythropoiesis by caspase-mediated cleavage of GATA-1. Nature 401:489-493

11. Ishizaki Y, Jacobson MD, Raff MC 1998 A role for caspases in lens fiber differentiation. J Cell Biol 140:153-158

12. Pandey P, Nakazawa A, Ito Y, Datta R, Kharbanda S, Kufe D 2000 Requirement for caspase activation in monocytic differentiation of myeloid leukemia cells. Oncogene 19:3941-3947

13. Huppertz B, Frank H-G, Kingdom JCP, Reister F, Kaufmann P 1998 Villous cytotrophoblast regulation of the syncytial apoptotic cascade in the human placenta. Histochem Cell Biol 110:495-508

14. Huppertz B, Frank H-G, Reister F, Kingdom J, Korr H, Kaufmann P 1999 Apoptosis cascade progresses during turnover of human trophoblast: analysis of villous cytotrophoblast and syncytial fragments in vitro. Lab Invest 79:1687-1702

15. Cirelli N, Lebrun P, Gueuning C, Moens A, Delonge-Desnoeck J, Dictus-Vermeulen C, Vanbellinghen AM, Meuris S 2000 Secretory characteristics and viability of human term placental tissue after overnight cold preservation. Hum Reprod 15:756761

16. Thornberry NA, Lazebnik Y 1998 Caspases: enemies within. Science 281:1312-1316

17. Fadeel B, Orrenius S, Zhivotovsky B 2000 The most unkindest cut of all: on the multiple roles of mammalian caspases. Leukemia 14:1514-1525

18. Kliman HJ, Nestler JE, Sermasi E, Sanger JM, Strauss JF III 1986 Purification, characterization and in vitro differentiation of cytotrophoblasts from human term placenta. Endocrinology 118:1567-1582

19. Thirkill TL, Douglas GC 1997 Differentiation of human trophoblast cells in vitro is inhibited by dimethylsulfoxide. J Cell Biochem 65:460-468

20. Douglas GC, King BF 1993 Colchicine inhibits human trophoblast differentiation in vitro. Placenta 14:187-201

21. Morrish DW, Bhardwaj D, Dabbagh LK, Marusyk H, Siy O 1987 Epidermal growth factor induces differentiation and secretion of human chorionic gonadotropin and placental lactogen in normal human placenta. J Clin Endocrinol Metab 65:1282-1290

22. Levitzki A, Gazit A 1995 Tyrosine kinase inhibition: an approach to drug development. Science 267:1782-1788

23. Ringler GE, Strauss JF III 1990 In vitro systems for the study of human placental endocrine function. Endocr Rev 11:105-123

24. Mayhew TM, Leach L, McGee R, Ismail WW, Myklebust R, Lammiman MJ 1999 Proliferation, differentiation and apoptosis in villous trophoblast at 13-41 weeks of gestation (including observations on annulate lamellae and nuclear pore complexes). Placenta 20:407-422

25. Lyden TW, Ng AK, Rote NS 1993 Modulation of phosphatidylserine epitope expression by BeWo cells during forskolin treatment. Placenta 14:177-186

26. Alsat E, Wyplosz P, Malassiné A, Guibourdenche J, Porquet D, Nessmann C, Evain-Brion D 1996 Hypoxia impairs cell fusion and differentiation process in human cytotrophoblast, in vitro. J Cell Physiol 168:346-353

27. Yusuf K, Smith SD, Levy R, Schaiff WT, Wyatt SM, Sadovsky Y, Nelson DM 2001 Thromboxane $\mathrm{A}_{2}$ limits differentiation and enhances apoptosis of cultured human trophoblasts. Pediatr Res 50:203-209

28. Redman CWG 1991 Current topic: Pre-eclampsia and the placenta. Placenta 12:301308

29. Cheng Y, Deshmukh MD, Costa A, Demaro JA, Gidday JM, Shah A, Sun Y, Jacquin MF, Johnson, Jr. EM, Holtzman DM 1998 Caspase inhibitor affords neuroprotection with delayed administration in a rat model of neonatal hypoxic-ischemic brain injury. J Clin Invest 101:1992-1999

30. Braun JS, Novak R, Herzog K-H, Bodner SM, Cleveland JL, Tuomanen EI 1999 Neuroprotection by a caspase inhibitor in acute bacterial meningitis. Nature Med $5: 298-302$ 